\title{
Reinforcement rate and immediacy of reinforcement as factors in choice ${ }^{1}$
}

Two pigeons were trained on a concurrent chain schedule. In the second links of the chains food was delivered on two fixed-interval schedules. For each chain the fixed intervals always summed to $60 \mathrm{sec}$, and the experimental conditions consisted of varying the fixed intervals on one key within this restriction. The results suggest that relative choice is determined by the duration of the first fixed intervals in the second links rather than by the reinforcement rates in the second links.

Experiments reported by Autor (1960) and Herrnstein (1964) have suggested that relative choice in concurrent chain schedules is controlled by the rate of reinforcement in the second links of the two concurrent chains. These studies have typically used VI or VR schedules in the second links and have neglected to control for the influence of the immediacy of primary reinforcement in the second links. On VI or VR schedules immediacy of reinforcement correlates very highly with overall reinforcement rate, and a recent experimentby Kendall (1967) has provided evidence that immediacy of reinforcement, rather than rate of reinforcement, is a controlling variable in similar but non-concurrent chained schedules. Kendall's procedure was to use a VI schedule in the conditionally reinforced first link after which food was produced on two FI schedules summing in duration to $120 \mathrm{sec}$. The fixed intervals were varied within this restriction, giving a constant overall rate of reinforcement, and it was found that the response rate in the VI link was mainly a function of the first fixed interval duration in the primarily reinforced links (PRLs).

The present experiment looks at the effects of immediacy of reinforcement in the PRLs on relative choice when overall reinforcement rates are similar in both chains. The chains used are similar to those used by Kendall (1967), with the VI links concurrent and the PRLs mutually exclusive.

Method

Two adult white Carneaux pigeons, maintained at $80 \%$ of their free feeding body weights, served as Ss. Reinforcement consisted of 3.5 sec access to mixed grain, and sessions were terminated after a fixed number of reinforcements had been gained.

Multiple stimulus projectors were used to transilluminate the outer two keys of a three-key pigeon chamber. Training sessions began with both keys transilluminated by red light. In this part of the procedure both keys were associated with independent VI schedules each with a mean interval of $60 \mathrm{sec}$, and a change-over delay of $0.5 \mathrm{sec}$ was in force to prevent excessive switching between keys. When one VI programmer set up, pecks to the associated key changed the color of that key from red to white, and changed the color of the other key to black. Pecks to the blackened key had no programmed consequence and during the time that the key was blackened the associated VI programmer was stopped. Pecks to the white key were now primarily reinforced on a sequence of two Fl schedules. For Key 1 this sequence always consisted of FI 30 sec followed by FI $30 \mathrm{sec}$. For Key 2, each experimental condition consisted of a different fixed sequence of two intervals within the restriction that they should sum to $60 \mathrm{sec}$.

After the second reinforcement under the white conditions on either key, the first links of the chains were reinstated and the VI programmers started. If it happened that both VI programmers had set up before the the PRLs were entered, only the programmer associated with the key that had been chosen on that trial was restarted, the other remaining set up.

For Bird $\mathrm{C}$ the first intervals in the PRLs on Key 2 were $10,15,30$ and $45 \mathrm{sec}$, and for Bird F they were $15,20,25$ and $30 \mathrm{sec}$. The value of the second FI on this key in the PRLs was that necessary to make the sum to $60 \mathrm{sec}$. The birds were run under each condition in a roughly randomized order until the relative response rates in the first links, and the absolute rates in all links, appeared stable over 10 days. This required from 30 to 50 daily training sessions for each experimental condition.

Results

The results of this experiment are shown in the upper graphs of Fig. 1. The relative response rate on Key 1 during the VI links is the number of responses made on this key divided by the total number of responses made during these links. Each point represents the mean relative rate of the last 10 days of each condition. The horizontal line at relative rate 0.5 is the function expected if the relative response rates in the concurrent VI links were controlled by the relative reinforcement rates during the PRLs. The relative rates for both animals deviate from this function in a way which suggests that the relative response rates might be controlled largely by the reinforcement rate contributions of only the first intervals of the PRLs. To check on this possibility the data were replotted in the lower graphs of Fig. 1 in which the abscissa is the relative reinforcement rate determined by taking only these links into account and ignoring the contribution of the second FIs. The diagonal lines show the function that would be ex- 
pected if these relative reinforcement rates were directly controlling the relative response rates in the first links.

Cumulative records showed typical FI patterns of responding in the PRLs under all experimental conditions.

\section{Discussion}

The results of the present experiment suggest that choice in the concurrent chain procedure may be controlled largely by the interval that elapses between entering the PRLs and obtaining the first food reinforcement. The assumption that it is the first FI, rather than the second, which controls choice is made on the basis of the direction of the effect in the light of previous results (Herrnstein, 1964; Kendall, 1967). These results thus extend the generality of Kendall's findings to concurrent chain procedures and further suggest that the results of Autor (1960) and Herrnstein (1964) may need reinterpretation in terms of relative im-

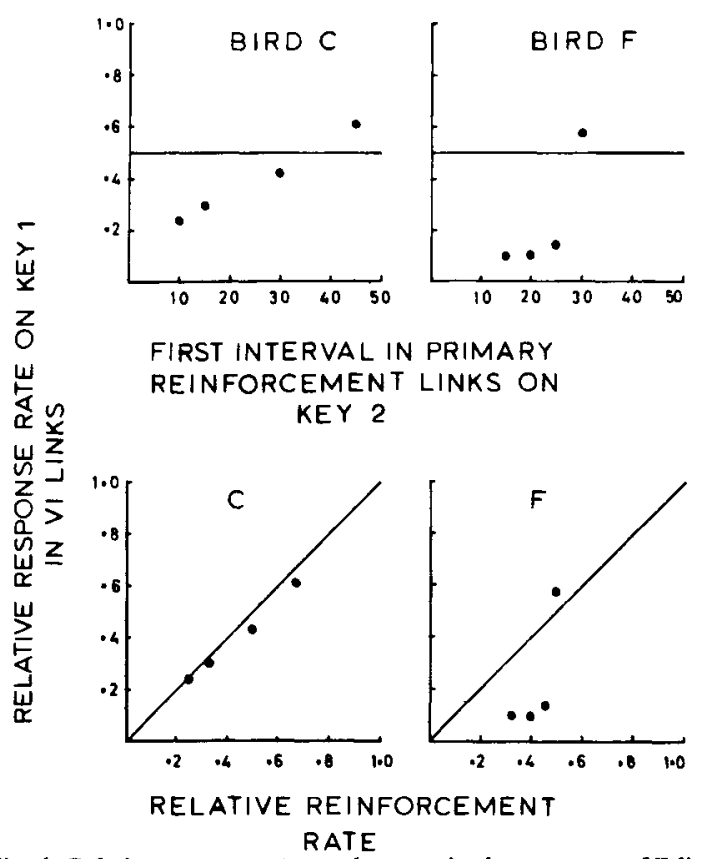

Fig. 1. Relative response rates on key one in the concurrent VI links as a function of the duration of the first FI in the PRLs on key two (upper); and as a function of the relative reinforcement rate provided by the first FIs in the PRLs (lower). mediacy of reinforcement, rather than relative rate of reinforcement, in the PRLs.

Since the major controlling variable appeared to be the first FI to food presentation, it could be that choice was being determined by the reinforcement rate contributions of only the first FIs in the PRLs. This function, in this procedure, is identical to the relative delay between the completion of the VI conditions on one key and primary reinforcement. Chung \& Herrnstein (1967) have shown that the relative response rate in concurrent VI schedules is a linear function of the programmed relative delay of reinforcement when an animal is choosing between different delay intervals. If a similar factor were controlling relative choice in the present experiment we should expect that the control exerted by the first FI would be modified somewhat by the second FI. From these considerations it would be expected that the data points in the lower graphs of Fig. 1 would fall above the diagonal when the relative reinforcement rate on Key 2 was below 0.5 and below the diagonal when it was above 0.5 . The results, by and large, indicate the opposite of this effect. For Bird $\mathrm{C}$ the data points fell close to the diagonal, allowing no modification by the second interval. For Bird $F$ the relative rates fell well below the diagonal when the relative reinforcement rate was below 0.5 . The present results are therefore not directly compatible with those of Chung \& Herrnstein (1967), and suggest that the amount of control over relative choice exerted by the second FI in the PRLs might be a function of both the duration of this interval and the duration of the first interval.

References

AUTOR, S. M. The strength of conditioned reinforcers as a function of frequency and probability of reinforcement. Unpublished doctoral dissertation, Harvard University, 1960.

CHUNG, SHIN-HO, \& HERRNSTEIN, R. J. Choice and delay of reinforcement. J. exp. Anal. Behav., 1967, 10, 67-74.

HERRNSTEIN, R. J. Secondary reinforcement and rate of primary reinforcement. J. exp. Anal. Behav., 1964, 7, 27-36.

KENDALL, S. B. Some effects of fixed-interval duration on response rate in a two component chain schedule. J. exp. Anal. Behav., $1967,10,341-347$.

Note

1. This study was carried out during the tenure of a Commonwealth Scholarship. I would like to thank Dr. Ray Over for his help and advice. 